\section{SOI: $1.1 /$ TAS $\quad$ DOI: $10.15863 /$ TAS International Scientific Journal Theoretical \& Applied Science}

\author{
Andrey Leonidovich Gusev \\ Doctor of Technical Sciences \\ professor of Perm State \\ National Research University \\ (PSU) \\ alguseval@mail.ru
}

Alexander Anatolevich Okunev
Post-graduate student of the Perm State
National Research University

(PSU)

SECTION 2. Applied Mathematics.

Math modeling.

\title{
NEURAL NETWORKS ABOUT MAN
}

Abstract: The article examines the possibility of determining the qualities of a person's personality (truthfulness / deceit, degree of intelligence and reliability) using neural networks, determining the degree of susceptibility of a person to various diseases (cancer, infarction), determining a person's inclination to a profession (dancer, mathematician, doctor, financier), to predict the duration of a person's life. Describes studies that were conducted using the exact time and place of birth of a person.

Key words: perceptron, neural network error, life expectancy, personality qualities, propensity to the profession, human susceptibility to diseases.

Language: Russian

Citation: Gusev AL, Okunev AA (2018) NEURAL NETWORKS ABOUT MAN. ISJ Theoretical \& Applied Science, 01 (57): 266-271.

Soi: http://s-o-i.org/1.1/TAS-01-57-43 Doi: crossef https://dx.doi.org/10.15863/TAS.2018.01.57.43

\section{НЕЙРОННЫЕ СЕТИ О ЧЕЛОВЕКЕ}

Аннотация: В статье рассматривается возможность с помощью нейронных сетей определять качества личности человека (правдивость/лживость; степени ума и надёжности), устанавливать степень подверженности человека различным заболеваниям (рак, инфаркт), определять у человека склонность к профессии (танцуощуик, математик, врач, финансист), прогнозировать продолжительность жизни человека. Описываются исследования, которые проводились с использованием точного времени и места рождения человека.

Ключевые слова: персептрон, ошибка нейронной сети, продолжительность жизни, качества личности, склонность к профессии, подверженность человека к заболеваниям.

\section{Введение}

Один из авторов настоящей статьи в своё время опубликовал ряд работ [1-4], которые были посвящены исследованиям человеческих качеств личности, подверженности человека заболеваниям, продолжительности жизни человека и некоторым другим аспектам. В этих статьях использовались идеи $[5 ; 6]$. Все исследования проводились с помощью нейронных сетей персептронного типа с использованием функциональной предобработки статистических данных [7] и методов, описанных в [8]. Настало время сделать некоторые обобщения и выводы с использованием идей о всеобщей связи различного рода информации [9; 10], именно этому, и посвящена настоящая работа.

Как известно из астрологии для точного времени и места рождения любого человека можно составить натальную карту (гороскоп). Натальная карта основана на построении градуированной окружности, на которую наносятся астрологические точки с указанием градуса окружности: планеты, дома и некоторые другие. Далее исследуя расположение астрологических точек, астролог делает свои выводы о человеке. Для выявления различных статистических закономерностей в качестве источника статистической информации был использован один из самых больших и надёжных банков астрологических данных некоммерческий астрологический статистический банк астролога Lois Rodden, далее банк LR по инициалам основательницы. Опишем кратко эксперименты и их результаты, которые были подробно описаны в [1-4]. 


\section{Эксперимент 1. Качество личности «УМ»}

Из банка LR были сформированы наблюдения. Каждое наблюдение состояло из 25 астрологических точек и пола человека (1 мужской и 0 - женский) - это входные данные для нейронной сети (всего 26 входов) и диагноза умный/неумный (1 - умный, 0 - неумный $)$ - это выходные данные для нейронной сети (1 выход). Из группы Exceptional mind (Исключительный ум или разум) банка данных LR были взяты 158 наблюдений пригодных для статистической обработки у людей, зарекомендовавших себя умными людьми. За образец ума были взяты известные люди такие как: Вуди Ален, Нильс Бор, Никола Коперник, Леонардо де Винчи, Чарльз Диккенс, Томас Эдисон, Альберт Эйнштейн, Энрико Ферми, Бобби Фишер, Беньамин Франклин, Зигмунд Фрейд, Галилей Галилео, Виктор Гюго, Эммануил Кант, Пётр Капица, Гарри Каспаров, Микеланджело Буонарроти, Исаак Ньютон, Фридрих Ницше, Мишель де Нострадамус, Эрвин Шрёдингер, Вильям Шекспир, Барух Спиноза, Рихард Штраус, Никола Тесла, Норберт Винер и другие. Из группы I.Q. low (Низкий I.Q.) были взяты 124 наблюдения пригодных для статистической обработки у людей, страдающих слабоумием. За образец слабоумных были взяты, например, такие известные люди как: Принц Виктор Альберт (член Британской королевской семьи; первый сын Эдуарда VII; рассеянный, неустойчивый и слабоумный), Король Франции Луи XVI (был слаб в характере и скуден мыслями; всеми презираем) и другие. Всего 282 наблюдения.

В результате обучения нейронная сеть всем умным предсказала оценку быть умными больше 0,5, а всем неумным предсказала оценку быть умными меньше 0,5. Следовательно, можно предположить, что при использовании этой нейронной сети для прогноза умственных способностей у вновь наблюдаемых людей прогноз нейронной сети будет верным. То есть, для людей, которые умны, оценка ума (диагноз или прогноз нейронной сети) будет более 0,5 , а для людей, которые неумны, оценка ума будет меньше 0,5.

\section{Эксперимент 2. Качество личности «ЛЖИВОСТЬ»}

Из банка LR были сформированы наблюдения, каждое из которых состояло из 25 астрологических точек и пола человека (1 мужской и 0 - женский) - это входные данные для нейронной сети (всего 26 входов) и диагноза лживый/правдивый $\quad\left(\begin{array}{lll}1 & - & \text { лживость/не }\end{array}\right.$ принципиальность, 0 принципиальность/правдивость) - это выходные данные для нейронной сети (1 выход). Из группы Liar / fraud (Лжец / мошенничество) банка данных LR были взяты 82 наблюдения пригодных для статистической обработки у людей, зарекомендовавших себя лжецами. Из группы Principled strongly (Сильно принципиальные) были взяты 158 наблюдений, которые были пригодны для статистической обработки. Наблюдения были взяты у людей, которые зарекомендовали себя правдивыми и принципиальными. Всего 240 наблюдений.

В результате обучения нейронная сеть всем лжецам предсказала оценку быть лжецами больше 0,5, а всем правдивым предсказала оценку быть лжецами меньше 0,5. Следовательно, можно предположить, что при использовании этой нейронной сети для прогноза лживый/правдивый у вновь наблюдаемых людей прогноз нейронной сети будет верным. То есть, для людей, которые лживы, оценка лживости (диагноз или прогноз нейронной сети) будет более 0,5 , а для людей, которые правдивы, оценка лживости будет меньше 0,5 .

\section{Эксперимент 3. Качество личности «НАДЁЖНОСТЬ»}

Из банка LR были сформированы наблюдения. Каждое наблюдение состояло из 25 астрологических точек и пола человека (1 мужской и 0 - женский) - это входные данные для нейронной сети (всего 26 входов) и диагноза надёжный/подлый (1 - надёжность, 0 - подлость) - это выходные данные для нейронной сети (1 выход). Из группы Personality robust (Надёжный) банка данных LR были взяты 82 наблюдения пригодных для статистической обработки $\mathrm{y}$ людей, которые зарекомендовали себя надёжными. Из группы Difficult / mean spirited (Подлый) были взяты 66 наблюдений у людей, которые зарекомендовали себя подлыми. Всего 148 наблюдений.

В результате нескольких попыток обучения нейронной сети, была построена нейронная сеть, которая всем надёжным людям предсказала оценку быть надёжными больше 0,5 , а всем подлым людям предсказала оценку быть надёжными меньше 0,5. Следовательно, можно предположить, что при использовании этой нейронной сети для прогноза надёжный/подлый у вновь наблюдаемых людей прогноз нейронной сети будет верным. То есть, для людей, которые надёжны, оценка надёжности (диагноз или прогноз нейронной сети) будет более 0,5 , а для людей, которые подлы, оценка надёжности будет меньше 0,5 .

Эксперимент 4. Склонность к заболеванию «РАК» 
Из банка LR были сформированы наблюдения, каждое из которых состояло из 25 астрологических точек и пола человека (1 мужской и 0 - женский) - это входные данные для нейронной сети (всего 26 входов) и подверженность раку (1 - был рак, 0 - не было рака) - это выходные данные для нейронной сети (1 выход). ). Из группы Long life more than 80 yrs (смерть после 80 лет) банка данных LR были взяты 182 наблюдения (у людей, проживших 95 лет и более лет и не имевших раковых заболеваний). Из группы Cancer (Рак) были взяты 935 наблюдений у людей, болевших раком. Всего 1117 наблюдений.

В результате нескольких попыток обучения нейронной сети (уже после опубликования [2]), была построена нейронная сеть, которая всем людям болевшим раком предсказала оценку заболеть раком больше 0,5 , а всем людям, не болевшим раком, предсказала оценку заболеть раком меньше 0,5. Следовательно, можно предположить, что при использовании этой нейронной сети для прогноза подверженности раковому заболеванию у вновь наблюдаемых людей прогноз нейронной сети будет верным. То есть, для людей, которые подвержены раковым заболеваниям, оценка заболеть раком (диагноз нейросети) будет более 0,5 , а для людей, которые не подвержены раковым заболеваниям, оценка заболеть раком будет меньше 0,5.

\section{Эксперимент 5. Склонность к заболеванию «ИНФАРКТ»}

Из банка LR были сформированы наблюдения. Каждое наблюдение состояло из 25 астрологических точек и пола человека (1 мужской и 0 - женский) - это входные данные для нейронной сети (всего 26 входов) и подверженность инфаркту (1 - был инфаркт, 0 не было инфаркта) - это выходные данные для нейронной сети (1 выход). Из группы Long life more than 80 yrs (смерть после 80 лет) банка данных LR были взяты 207 наблюдения (у людей, проживших 95 лет и более лет и не имевших инфаркта). Из группы Heart disease / attack (Сердечнососудистые заболевания / атака (инфаркт)) были взяты 185 наблюдений у людей, подвергавшихся атаке на сердце (инфаркту). Всего 392 наблюдения.

В результате нескольких попыток обучения нейронной сети (уже после опубликования [2]), была построена нейронная сеть, которая всем людям, перенесшим инфаркт, предсказала оценку подверженности инфаркту больше 0,5, а всем людям, не имевшим инфаркта, предсказала оценку подверженности инфаркту меньше 0,5. Следовательно, можно предположить, что при использовании этой нейронной сети для прогноза подверженности инфаркту у вновь наблюдаемых людей прогноз нейронной сети будет верным. То есть, для людей, которые подвержены инфаркту, оценка перенести инфаркт (диагноз нейросети) будет более 0,5 , а для людей, которые не подвержены инфаркту, такая оценка будет меньше 0,5.

\section{Эксперимент 6. Склонность к профессиям «Танцовщик» и «Математик»}

Из банка LR были сформированы наблюдения, каждое из которых состояло из 27 астрологических точек и пола человека (1 мужской и 0 - женский) - это входные данные для нейронной сети (всего 28 входов) и диагноза: пригоден/непригоден к профессии (1 - пригоден, 0 - непригоден) - это выходные данные для нейронной сети (1 выход). Из группы Dancer/Teacher (Танцовщик/Хореограф) банка данных LR были взяты 76 наблюдений пригодных для статистической обработки $\mathrm{y}$ людей, зарекомендовавших себя в мире балета. За образец таких людей были взяты известные люди такие как: Георгий Баланчин, Михаил Барышников, Айсидора Дункан, Александр Годунов, Анна Павлова, Марис Петипа, Мата Хари и другие. Из группы Mathematics/Statistics (Математика/Статистика) были взяты 82 наблюдения пригодных для статистической обработки у людей, зарекомендовавших себя в мире математики. За образец таких людей были взяты известные люди такие как: Георг Кантор, Георг Буль, Ленхард Эйлер, Карл Фридрих Гаусс, Вильям Гамельтон, Давид Гильберт, Генри Пуанкаре, Якоби, Парето, Паскаль и другие. Всего 158 наблюдений.

В этом эксперименте, как и в следующих двух экспериментах, будем объединять «не сочетаемые» группы. Поясним это. Склонность к балетному искусству редко сочетается со склонностью к точным наукам (например, к математике), а также со склонностью к финансовому делу или медицине. Поэтому «не сочетаемыми» профессиями можно считать «танцовщик и математик», «танцовщик и финансист» и «танцовщик и врач».

В результате была построена нейронная сеть, которая всем людям из мира балета предсказала оценку склонности к профессии танцовщика или хореографа больше 0,5 , а всем математикам предсказала оценку быть танцовщиками меньше 0,5. Если рассмотреть разность между единицей и оценкой склонности к профессии, данной нейронной сетью, то эту разность можно интерпретировать как оценку склонности к математике. Чем ближе эта 
разность к единице, тем больше оценка склонности к профессии математики.

Интерпретация результата. Можно предположить, что при использовании этой нейронной сети для оценки склонности человека к профессии танцовщика/хореографа, а также к профессии математика у вновь наблюдаемых людей прогноз нейронной сети будет верным. То есть, для людей, которые склонны к балету, оценка склонности будет

более 0,5 , а для людей, которые склонны к математике, оценка будет меньше 0,5.

\section{Эксперимент 7. Склонность к профессиям «Танцовщик» и «Врач»}

В условиях эксперимента 6 наблюдения из группы «Математики» были заменены наблюдениями из группы «Врачи». А именно, из группы Physician (Врач) были взяты 39 наблюдений пригодных для статистической обработки у людей, зарекомендовавших себя в мире медицины. Таким образом, в этом эксперименте всего 115 наблюдений.

В результате эксперимента была построена нейронная сеть, которая всем людям из мира балета предсказала оценку склонности к профессии танцовщика или хореографа больше 0,5, а всем врачам предсказала оценку быть танцовщиками меньше 0,5. Если рассмотреть разность между единицей и оценкой склонности к профессии, данной нейронной сетью, то эту разность можно интерпретировать как оценку склонности к медицине. Чем ближе эта разность к единице, тем больше оценка склонности к профессии врача-медика.

Интерпретация результата. Можно предположить, что при использовании этой нейронной сети для оценки склонности человека к профессии танцовщика/хореографа, а также к профессии врача у вновь наблюдаемых людей прогноз нейронной сети будет верным. То есть, для людей, которые склонны к балету, оценка склонности будет более 0,5 , а для людей, которые склонны к медицине, оценка будет меньше 0,5 .

\section{Эксперимент 8. Склонность к профессиям «Танцовщик» и «Финансист»}

В условиях эксперимента 6 наблюдения из группы «Математики» были заменены наблюдениями из группы «Финансисты». А именно, из группы Banker/Financier (Банкир/Финансист) были взяты 30 наблюдений пригодных для статистической обработки у людей, зарекомендовавших себя в мире финансов. Таким образом, в этом эксперименте всего 106 наблюдений.
В результате эксперимента была построена нейронная сеть, которая всем людям из мира балета предсказала оценку склонности к профессии танцовщика или хореографа больше 0,5 , а всем финансистам предсказала оценку быть танцовщиками меньше 0,5. Если рассмотреть разность между единицей и оценкой склонности к профессии, данной нейронной сетью, то эту разность можно интерпретировать как оценку склонности к финансовому делу. Чем ближе эта разность к единице, тем больше оценка склонности к профессии банкира-финансиста.

Интерпретация результата. Можно предположить, что при использовании этой нейронной сети для оценки склонности человека к профессии танцовщика/хореографа, а также к профессии финансиста у вновь наблюдаемых людей прогноз нейронной сети будет верным. То есть, для людей, которые склонны к балету, оценка склонности нейронной сети будет более 0,5, а для людей, которые склонны к финансовому делу, оценка будет меньше 0,5. жизни

Эксперимент 9. Продолжительность

Из банка LR были сформированы наблюдения. Каждое наблюдение состояло из 25 астрологических точек и пола человека (1 мужской и 0 - женский) - это входные данные для нейронной сети (всего 26 входов) и количество прожитых лет - это выходные данные для нейронной сети (1 выход). Из группы Long life more than 80 yrs (смерть после 80 лет) были отобраны по соображениям готовности к статистической обработке 1490 наблюдений.

Заранее определимся, что будем считать ошибкой нейронной сети при прогнозе. Для конкретного наблюдения есть точное значение количества прожитых лет человеком и количество лет прожитых человеком, рассчитанные (предсказанные или прогнозируемые) нейронной сетью. Модуль разницы этих значений, деленный на количество лет прожитых человеком, и умноженный на $100 \%$ будем считать ошибкой нейронной сети для этого наблюдения. Если наблюдения объединены в группу, то среднее значение ошибки по группе для наблюдений, входящих в группу, будем считать ошибкой нейронной сети для группы наблюдений.

Для эксперимента все 1490 наблюдений были разделены произвольным образом на три части: обучающее множество - 1192 наблюдения (80\% всех наблюдений), тестирующее множество - 149 наблюдений (10\% всех наблюдений) и подтверждающее множество - 149 наблюдений (10\% всех наблюдений). 
В результате нескольких попыток была построена нейронная сеть, которая имела ошибку для обучающего множества равную 4,9\%, ошибку для тестирующего множества равную $5,18 \%$ и ошибку для подтверждающего множества равную 5,17\%. Таким образом, общая ошибка для всех 1490 наблюдений равнялась $4,96 \%$.

Интерпретация результата. Так как в эксперименте участвовали наблюдения только из группы Long life more than 80 yrs (смерть после 80 лет), то можно утверждать, что для людей переживших 80-летний рубеж нейронная сеть может определить продолжительность жизни в среднем с точностью до 5\% (ошибка нейронной сети). А если прогноз нейронной сети интерпретировать как максимально возможную продолжительность жизни для любого человека (в не зависимости от того, перешагнёт он или нет 80-летний рубеж), то ошибка нейронной сети будет ещё меньше по очевидным причинам.

\section{Эксперимент 10. Предел точности прогноза нейронной сети для продолжительности жизни человека}

Из банка LR к наблюдениям предыдущего эксперимента добавили из категории Accidental (смерть от случая) - 131 наблюдение; из категории Disease (смерть от болезни) - 324 наблюдения; из категории Short Life less than 29 yrs (смерть до 29 лет) - 315 наблюдений; из категории Suicide (самоубийство) - 195 наблюдений; из категории Unusual (необычная смерть) - 74 наблюдения; из категории Other Death (остальные смерти) - 31 наблюдение. Таким образом, получили множество из 2560 наблюдений.

Далее построили нейросеть на всех 2560 наблюдениях без разделения на обучающее, тестирующее и подтверждающее множества. Обучали нейросеть до тех пор, пока ошибка не прекратила существенно снижаться (примерно 4000 итераций). Далее нашли ошибку для каждого наблюдения. Выделили из всех наблюдений те (аномальные наблюдения), у которых ошибка больше $25 \%$ и реальная продолжительность жизни, зафиксированная у этих наблюдений, меньше 10 лет. Таких наблюдений оказалось 39 из 2560 наблюдений, что составило $1,52 \%$ от всех исследуемых наблюдений.

Установлено, что для аномальных наблюдений средняя ошибка составила менее трёх лет. Причём такая средняя ошибка выявлена для детей возраста младше 10 лет, а детская смертность является, как известно, наименее прогнозируемой и предсказуемой категорией.

Теперь рассмотрим остальные наблюдения (без аномальных), средняя продолжительность жизни которых составляет 70,2 года. Здесь средняя ошибка зафиксирована на уровне 0,997\%. Следовательно, результат каждого прогноза нейронной сети отличался от реальной продолжительности жизни в среднем на 0,7 года. Следовательно, такой предел точности прогноза нейронной сети для общей продолжительности жизни человека.

Однако не следует заблуждаться в том, что если бы, например, множество из 2560 наблюдений были разделены на три множества: обучающее, тестирующее и подтверждающее, то результат был бы похож на результат 9 эксперимента. По мнению экспертов можно предположить, что для построения «хорошей» нейронной сети с результирующей ошибкой до 1 года в этом эксперименте, необходимо порядка 30000-35000 наблюдений, взятых приблизительно в равных количествах из каждой категории.

\section{Заключение}

Первый вывод - нейронные сети хорошо «улавливают» закономерности влияния астрологических точек натальной карты:

- на качества личности: ум, лживость и надёжность,

- на подверженность человека к заболеваниям раком и инфарктом,

- на склонность человека к профессиям: танцовщик/хореограф, математик, финансист и медик-врач,

- на общую продолжительность жизни человека.

Отсюда следует полагать, что нейросеть «уловит» закономерности влияния астрологических точек на различные качества личности, на подверженность всевозможным заболеваниям, на склонность человека к разнообразным профессиям.

Второй вывод - для того чтобы прогноз нейросети во всех экспериментах был как можно точнее, необходимо увеличивать количество наблюдений из всех категорий, которые присутствуют в экспериментах, то есть «показывать» нейронной сети весь спектр категорий, каждая из которых должна быть максимально представительна. 


\begin{tabular}{l|lr|ll|ll} 
& ISRA (India) & $=\mathbf{1 . 3 4 4}$ & SIS (USA) & $=\mathbf{0 . 9 1 2}$ & ICV (Poland) & $=\mathbf{6 . 6 3 0}$ \\
Impact Factor: & ISI (Dubai, UAE) $=\mathbf{0 . 8 2 9}$ & PUHL (Russia) $=\mathbf{0 . 2 0 7}$ & PIF (India) & $=\mathbf{1 . 9 4 0}$ \\
& GIF (Australia) & $\mathbf{0 . 5 6 4}$ & ESJI (KZ) & $=\mathbf{4 . 1 0 2}$ & IBI (India) & $\mathbf{= 4 . 2 6 0}$ \\
& JIF & $\mathbf{1 . 5 0 0}$ & SJIF (Morocco) $=\mathbf{2 . 0 3 1}$ & & \\
\hline
\end{tabular}

\section{References:}

1. Gusev A.L., Yasnitsky L.N. (2015) Neural Networks and Lifespan: Eastern European Scientific Journal, 2015, №. 4; 188-194.

2. Gusev A.L. (2015) Neural Networks and Personality Traits: Eastern European Scientific Journal, 2015, №. 5; 99-108.

3. Gusev A.L. (2015) Neural Networks and the Propensity for the Profession: Eastern European Scientific Journal, 2015, №. 5; 109-112.

4. Gusev A.L., Yasnitsky L.N. (2017) Astrology under the prism of neural networks: Symvol nauky, 2017, №3. T. 3, p. 17-24.

5. Gauquelin M. (1991) Neo-Astrology: A Copernican Revolution. London: Arkana, Penguin Group.

6. Kefer Ya. (1993) Practical astrology, or the art of foresight and opposition to fate: in 5 books. Saratov: MP "Hope", 1993; v.1, 264 p.; v.2, 296 p.
7. Gusev A.L, Cherepanov F.M, Yasnitsky L.N. (2013) Functional preprocessing of input signals of a neural network. Neurocomputers: development, application. 2013. № 5. -p. 19-21.

8. Gusev A.L., Okunev A.A. (2017) Forecasting with incomplete set of factors determining the predicted factor. Neural network error extrapolation method. International Journal of Applied Mathematics and Statistics. 2017, Vol.56, №5, -p.48-52.

9. Wiener N. (1983) Cybernetics, or Control and Communication in Animal and Machine. Moscow: Science; The main edition of publications for foreign countries, 1983. - 344 p.

10. Gusev A.L. (2017) Mathematical analogue of the Universe model. Symvol nauky, 2017, №3. T. 3, -p. 11-17. 